\title{
Chronic Exposure to Diesel Exhaust Particulate Matter Impairs Meiotic Progression during Spermatogenesis in a Mouse Model
}

\section{Wei Yang}

Fudan University School of Public Health

\section{Yanyi Xu}

Fudan University School of Public Health

\section{Hongjie Pan}

Shanghai Institute of Planned Parenthood Research

\section{Fang Tian}

Shanghai Institute of Planned Parenthood Research

\section{Yuzhu Wang}

Shanghai Institute of Planned Parenthood Research

\section{Minjie Xia}

Shanghai Institute of Planned Parenthood Research

Jingying Hu

Shanghai Institute of Planned Parenthood Research

\section{Mingjun Yang}

Shanghai Institute of Planned Parenthood Research

\section{Shimin Tao}

Fudan University School of Pharmacy

\section{Shenfei Sun}

Shanghai Institute of Planned Parenthood Research

\section{Haidong Kan}

Fudan University School of Public Health

\section{Runsheng Li}

Shanghai Institute of Planned Parenthood Research

\section{Zhekang Ying}

University of Maryland School of Medicine

Weihua Li ( $\nabla$ weihua.li@sippr.org.cn )

Fudan University School of Public Health 
Keywords: air pollution, diesel exhaust PM2.5, spermatogenesis, meiotic arrest

Posted Date: January 2nd, 2020

DOl: https://doi.org/10.21203/rs.2.19909/v1

License: (c) (1) This work is licensed under a Creative Commons Attribution 4.0 International License. Read Full License

Version of Record: A version of this preprint was published at Ecotoxicology and Environmental Safety on October 1st, 2020. See the published version at https://doi.org/10.1016/j.ecoenv.2020.110881. 


\section{Chronic Exposure to Diesel Exhaust Particulate Matter Impairs Meiotic}

\section{Progression during Spermatogenesis in a Mouse Model}

Wei Yang ${ }^{1,{ }^{*}}$, Yanyi $\mathrm{Xu}^{2,{ }^{*}}$, Hongjie Pan ${ }^{1,{ }^{*}}$, Fang Tian ${ }^{1}$, Yuzhu Wang ${ }^{1}$, Minjie Xia ${ }^{1}$, Jingying $\mathrm{Hu}^{1}$, Mingjun Yang ${ }^{1}$, Shimin Tao ${ }^{1}$, Shenfei Sun ${ }^{1}$, Haidong Kan², Runsheng $\mathrm{Li}^{1 \text {,\#}, \text { Zhekang Ying }}{ }^{3, \#}$, Weihua Li ${ }^{1, \#}$

${ }^{1} \mathrm{NHC}$ Key Lab. of Reproduction Regulation (Shanghai Institute of Planned Parenthood Research), School of Public Health, Fudan University, Shanghai, China.

${ }^{2}$ Department of Environmental Health, School of Public Health, Fudan University, Shanghai 200032, China

${ }^{3}$ Department of Medicine Cardiology Division, University of Maryland School of Medicine, Baltimore, Maryland 21201, USA

*These authors contributed equally to this work

Wei Yang: 17211150007@fudan.edu.cn; Yanyi Xu: yanyi xu@fudan.edu.cn; Hongjie Pan: phiie1994@163.com; Fang Tian: fannietian@qq.com; Yuzhu Wang: 466959008@qq.com; Minjie Xia: mito1983@163.com; Jingying Hu: hujingying@aliyun.com; Mingjun Yang: mingjun0707@163.com; Shimin Tao: 18111150003@fudan.edu.cn; Shenfei Sun: sunshenfei@tom.com; Haidong Kan: kanh@fudan.edu.cn; Runsheng Li: runshengli2007@163.com; Zhekang Ying: yingzhekang@hotmail.com; Weihua Li: weihua.li@sippr.org.cn

\#Address for Correspondence:

Runsheng Li, Ph.D.

4 Department of Reproductive Biology 
25 NHC Key Lab. of Reproduction Regulation

26 Shanghai Institute of Planned Parenthood Research

27 Institute of Reproduction and Development, Fudan University

282140 Xie Tu Road, Shanghai 200032, P.R.China

29 E-mail: runshengli2007@163.com

$30 \quad$ Tel: $81-21-64437446$

31 Fax: 81-21-64046128

32 Or

33 Zhekang Ying, Ph.D.

34 Department of Medicine Cardiology Division, School of Medicine,

35 University of Maryland

3620 Penn St. HSFII S005, Baltimore, MD 21201, USA

37 Email: yingzhekang@hotmail.com

$38 \quad$ Tel: $410-706-3586$

39 Or

40 Weihua Li, Ph.D.

41 Department of Pharmacology and Toxicology

42 NHC Key Lab. of Reproduction Regulation

43 Shanghai Institute of Planned Parenthood Research

44 Institute of Reproduction and Development, Fudan University

452140 Xie Tu Road, Shanghai 200032, P.R.China

$46 \quad$ E-mail: weihua.li@sippr.org.cn

$47 \quad$ Tel: $81-21-64437475$

$48 \quad$ Fax: 81-21-64046128

49 


\section{Abstract}

51 Background: Exposure to air pollutants represented by diesel exhaust $\mathrm{PM}_{2.5}$ (DEP) correlates 52 with the decline of semen quality, but the underlying biological mechanism has not been fully 53 understood. In the present study, mice were intratracheally instilled with DEP for around 7 54 months, and the effects of $\mathrm{PM}_{2.5}$ exposure on the spermatogenic process as well as the 55 alterations of testicular gene expression profile were assessed.

56 Results: Our results show that chronic exposure to DEP significantly impairs the fertility of male 57 mice without influencing their libido. Compared with Vehicle-exposed group, the sperm count 58 and motility from DEP-exposed mice were significantly decreased. In addition, 59 immunohistological staining of $\mathrm{yH} 2 \mathrm{AX}$ and $\mathrm{DMC} 1$, biomarkers for meiotic double strand breaks 60 (DSBs), demonstrated that chronic exposure to DEP comprised the repair of meiotic DSBs, thus 61 disrupts the spermatogenesis. Deep RNA sequencing test shows massive altered expressions 62 of testicular genes including the $\mathrm{GnRH}$ signaling pathway.

63 Conclusion: In summary, our research demonstrates that chronic exposure to $\mathrm{PM}_{2.5}$ disrupts 64 spermatogenesis through targeting the meiotic recombination, providing a new perspective for 65 the research on the male reproductive system damage caused by air pollution.

67 Key Words: air pollution; diesel exhaust $\mathrm{PM}_{2.5}$; spermatogenesis; meiotic arrest 
70 Epidemiological studies demonstrate a global uncontrolled decline in the semen quality and

71 male fertility rate over the past several decades [1,2]. The reasons for this decline has not yet

72 been well established. As this is a global decline, genetics apparently may not be its main

73 reasons. The male reproductive system is well known to be vulnerable to various environmental

74 stressors. Thus, environmental pollution is believed to probably account for the major decline in

75 the quality of semen. Ambient fine particulate matter $\left(\mathrm{PM}_{2.5}\right)$ is an airborne pollutant that severely threats the global public health. It is estimated that $91 \%$ of the world's population lives in places where air quality exceeds the world health organization (WHO) guideline limits (who.int/airpollution/en). Furthermore, $\mathrm{PM}_{2.5}$ levels markedly below the WHO guideline limit may still be harmful to public health [3]. Therefore, any adverse health effect of $\mathrm{PM}_{2.5}$ may be a significant threat for the global public health. Notably, epidemiological studies have increasingly shown that exposure to $\mathrm{PM}_{2.5}$ correlates with decline in semen quality [4-8], drawing considerable attention to the role of $\mathrm{PM}_{2.5}$ pollution in the global increase in male infertility.

Studies in animal models are essential to establish the causal role of $\mathrm{PM}_{2.5}$ exposure in the development of male infertility. Since Watanabe and Oonuki showed that inhalation of diesel engine exhaust affects spermatogenesis in growing male rats in 1999 [9], rapidly increasing studies in animal models have also demonstrated various adverse effects of $\mathrm{PM}_{2.5}$ exposure on semen quality. The sperm count, motility, and morphology are three most frequently used indexes for assessing the quality of semen. To date, studies in various animal models all demonstrate a decrease in the sperm count and motility, if assessed [9-18]. In contrast, both

90 negative $[11,14,16-18]$ results have been reported regarding the effect of $\mathrm{PM}_{2.5}$ exposure on the

91 rate of morphologically abnormal sperm. The integrity of the blood-testis barrier (BTB) is 
essential for spermatogenesis. Several studies show that exposure to $\mathrm{PM}_{2.5}$ results in a disruption of the BTB $[10-13,16]$, providing a potential mechanism for the spermatogenic abnormalities induced by $\mathrm{PM}_{2.5}$ exposure. In addition, exposure to $\mathrm{PM}_{2.5}$ was shown to evoke reactive oxygen species (ROS) production [12,17], inflammation [18], and endoplasmic reticulum (ER) stress [14] in the testes, suggesting that these may also be local mediators for the spermatogenic abnormalities induced by $\mathrm{PM}_{2.5}$ exposure.

Given that the major components of inhaled $\mathrm{PM}_{2.5}$ may not enter the systemic circulation, a mechanism linking $\mathrm{PM}_{2.5}$ inhalation to the pathology in the testis is clearly needed. Nanoparticles and polycyclic aromatic hydrocarbons (PAHs) in $\mathrm{PM}_{2.5}$ may enter the systemic circulation with various efficiencies. Notably, both nanoparticles [19] and PAHs [20] were shown to disrupt testicular structure and/or function, supporting that the adverse testicular effects of $\mathrm{PM}_{2.5}$ exposure may be mediated by egress of inhaled $\mathrm{PM}_{2.5}$. However, the high doses used in these studies somehow undermine this possibility. The hypothalamic-pituitary-gonadal (HPG) axis, including gonadotropin-releasing hormone $(\mathrm{GnRH})$ from the hypothalamus, luteinizing hormone $(\mathrm{LH})$ and follicle-stimulating hormone $(\mathrm{FSH})$ from the anterior portion of the pituitary gland, and estrogen and testosterone produced by the gonads, is central in maintaining the homeostasis of the male reproductive system. Watanabe and Oonuki showed that inhalation of diesel engine exhaust significantly affects the circulating levels of $\mathrm{FSH}$, LH, testosterone, and estradiol in rats [9]. We recently demonstrated that exposure to concentrated ambient $\mathrm{PM}_{2.5}$ (CAP) influences not only circulating $\mathrm{FSH}$ and testosterone but also the hypothalamic expression of $\mathrm{GnRH}$ [15]. These studies strongly suggest that the HPG axis may be a crucial mediator for those adverse testicular effects of $\mathrm{PM}_{2.5}$ exposure.

Spermatogenesis is a complicated process that includes the mitotic division that produces type A (self-renewal of stem cells) or B (committed to spermatocytes) spermatogonia, the 
116 meiotic division that produces haploid spermatids, and the spermiogenesis that transforms 117 spermatids into sperms. Notably, despite the above-mentioned considerable evidence for the 118 disruption of spermatogenesis by PM2.5 exposure, how PM2.5 exposure affects the 119 spermatogenic process has hardly been investigated, except for the histological analysis of 120 seminiferous tubules $[9,15]$. Therefore, the present study exploits the mouse model of 121 intratracheal instillation of diesel exhaust PM2.5 (DEP) to examine the effects of PM2.5 122 exposure on the spermatogenic process and also thoroughly document the alterations of 123 testicular gene expression profile induced by DEP exposure. Our results show that DEP 124 exposure decreased the number of advanced spermatogenic cells but not spermatogonia, 125 paralleled by marked increase in meiotic double strand breaks (DSBs) in pachytene but not 126 leptotene spermatocytes, strongly suggesting that DEP exposure disrupts spermatogenesis 127 through specifically targeting the repair of meiotic DSBs. 


\section{Results}

\section{Chronic exposure to DEP impairs the male fertility}

131 To examine the effect of chronic DEP exposure on the fertility of males, male C57BI/6J mice 132 were subject to 6-month intratracheal instillation of vehicle (PBS) or DEP, and then their 133 fertilities were assessed via an 18-day mating with normal female C57BI/6J mice. As shown in

134 Figure 1A, these sires were continued with their intratracheal instillation of vehicle or DEP 135 throughout this 18-day mating and the following 1-week singly housing. Therefore, they had 136 been exposed to vehicle or DEP for approximately 7 months in total when euthanized. Figures 137 1B and 1C show that all the sires inseminated their dam during this 18-day mating and that the 138 times taken for Vehicle- or DEP-exposed sires to inseminate their dam were comparable, 139 suggesting that exposure to DEP may not impact the libido of male mice. In contrast, Figure 1D 140 reveals that while $100 \%$ Vehicle-exposed sires impregnated their dam during this 18 -day mating, $14170 \%$ DEP-exposed sires only impregnated their dam, revealing that DEP exposure markedly 142 impairs the fertility of male mice. All the dams were sacrificed on day E16.5. The outcomes of 143 these pregnancies are presented in Table 1. The paternal exposure to DEP did not significantly 144 influence the rates of stillbirth and absorption, implantations per dam, live fetuses per dam, live 145 fetus weight, placental weight and uterus weight.

\section{Chronic exposure to DEP impairs the quality of semen}

148 Given the impairment of fertility of male mice by DEP exposure, we further documented the

149 effect of DEP exposure on the male reproductive system. Table 2 reveals that this 6-month 150 exposure to DEP did not significantly influence the weights of mouse body, testis, and 151 epididymis. In contrast, it significantly reduced the count of epididymal sperms (Figure 2B) and 
152 significantly decreased their motility (Figure 2C), strongly suggesting that chronic exposure to

153 DEP disturbs the development and maturation of sperms. However, the morphological analysis

154 of epididymal sperms showed that chronic exposure to DEP did not significantly influence the 155 rate of morphologically abnormal sperms (Figures 2A and 2D).

\section{Chronic exposure to DEP alters the testicular histology}

158 The testis is the organ that produces sperms. Given the reduction in the epididymal sperm count 159 by chronic exposure to DEP, histological analyses were performed on the testes of Vehicle- or 160 DEP-exposed mice to assess the effects of chronic DEP exposure on testicular histology. In 161 agreement with the reduced epididymal sperm count by chronic DEP exposure (Figure 2B), we 162 observed significant increases in Sertoli cell vacuolization (Figures 3A and 3B) and 163 derangement of the cell layers of seminiferous tubules (Figures $3 \mathrm{~A}$ and $3 \mathrm{C}$ ) in the testes of 164 DEP-exposed mice versus those of vehicle-exposed mice. Sperms are released in Stage VIII 165 seminiferous tubules; therefore, the proportion of Stage VIII seminiferous tubules somehow 166 represents the sperm production rate. We thus assessed the proportion of Stage VIII 167 seminiferous tubules in these testes. In line with the reduction in the epididymal sperm count by 168 chronic DEP exposure, Figure 3D reveals that DEP- versus Vehicle-exposed mice had 169 significantly reduced proportion of Stage VIII seminiferous tubules in the testes. In contrast, 170 chronic exposure to DEP did not significantly alter the proportion of Stage VII seminiferous 171 tubules (Figure 3E), the most frequently used seminiferous tubules for assessing the 172 spermatogenic parameters. 

seminiferous tubules

176 To pinpoint the effects of chronic exposure to DEP on spermatogenesis, we further documented 177 the spermatogenetic parameters of the Stage VII seminiferous tubules. Figures $4 \mathrm{~A}-4 \mathrm{C}$ show 178 that chronic exposure to DEP did not significantly affect the wall thickness, diameter, and Sertoli 179 cell number of the Stage VII seminiferous tubules. Notably, chronic exposure to DEP 180 significantly reduced the number of total germ cells in the Stage VII seminiferous tubules 181 (Figure 4D). Cell differentiation analysis demonstrated that chronic exposure to DEP did not 182 significantly change the number of spermatogonia (Figure 4E) but significantly reduced the 183 numbers of advanced spermatogenic cells including the pachytene spermatocytes (Figure 4F) 184 and round spermatids (Figure $\mathbf{4 G}$ ) in the Stage VII seminiferous tubules.

\section{Chronic exposure to DEP impairs the repair of meiotic double strand breaks (DSBs)}

187 During the spermatogenesis, the spermatogonia produces the primary spermatocyte through 188 mitosis, and the advanced spermatogenic cells are in turn produced by the primary 189 spermatocyte through meiosis. Therefore, the chronic DEP exposure-induced loss of advanced 190 spermatogenic cells strongly suggests that chronic DEP exposure disrupts the meiotic 191 progression during spermatogenesis. A major event during meiosis is the reshuffling of the 192 parental genomes through the formation and repair of DSBs, and there is a surveillance 193 mechanism called the recombination checkpoint ensures that all breaks are repaired before a 194 cell starts the meiotic divisions. To determine the effect of chronic DEP exposure on the 195 formation and repair of DSBs during meiosis, the spermatocyte spreads were prepared from the 196 testes of these Vehicle- or DEP-exposed mice and their DSBs were visualized using SYCP3 
197 and $\mathrm{yH} 2 \mathrm{AX}$ antibodies. Figures 5A-5D demonstrate that chronic DEP exposure did not 198 influence the distribution pattern of $\mathrm{yH} 2 \mathrm{AX}$, the biomarker for meiotic DSBs, in the leptotene and 199 zygotene spermatocytes, but significantly increased the rate of abnormal XY body and other 200 homologous chromosome in the pachytene spermatocytes, indicators for abnormal repair of 201 DSBs. For further analysis, we then staged and examined spermatocyte spreads by immuno202 localization of SYCP3/ $\mathrm{YH} 2 \mathrm{AX}$, which were used as the markers of different stages of meiotic 203 prophase. As shown in Figure 5E, the proportion of spermatocytes in leptotene and zygotene 204 stages in the DEP group increased, while the proportion of those in pachytene and diplotene 205 stages decreased, strongly suggesting that DEP severely interfered with the progression of 206 meiotic prophase.

207 To verify the effect of chronic DEP exposure on the repair of meiotic DSBs, the spermatocyte 208 spreads were also stained with DMC1, another biomarker for DSBs. Figures $\mathbf{5 F}-\mathbf{5 H}$ reveal that chronic exposure to DEP did not influence the number of DSBs in zygotene spermatocytes but 210 significantly increased the number of DSBs in pachytene spermatocytes, corroborating that 211 chronic DEP exposure does not impact the formation of DSBs but impairs their repair and thus 212 may delay the meiotic progression.

213 To verify whether chronic exposure to DEP disrupts the spermatogenesis through impact on 214 the repair of DSBs, the sections of testes from the Vehicle- and DEP-exposed mice were 215 visualized using $\mathrm{yH} 2 \mathrm{AX}$ antibody. Figure $6 \mathrm{~A}$ shows that the $\mathrm{yH} 2 \mathrm{AX}^{+}$cells in the advanced 216 spermatocytes (cells close to the lumen of seminiferous tubules) were markedly increased in the 217 testes of DEP-exposed mice versus those of Vehicle-exposed mice, strongly supporting the 218 impairment of repair of DSBs and thus disruption of the meiotic progression by chronic DEP 219 exposure. In normal testes such as the PBS-exposed in Figure 6A, most $\mathrm{yH}_{2} \mathrm{AX} \mathrm{X}^{+}$cells are the 220 early spermatogenic cells (those close to the base membrane), and the visualization of $\mathrm{yH} 2 \mathrm{AX}$ 
221 facilitates determining the meiotic stages of these early spermatogenic cells: the diffused

222 distribution pattern marked by the yellow arrows in Figure 6A represents the leptotene or 223 zygotene spermatocyte; and the focused distribution pattern marked by the red arrows in Figure 224 6A represents the pathytene or diplotene spermatocyte. Therefore, we analyzed the meiotic 225 stages of seminiferous tubules using the $\mathrm{yH} 2 \mathrm{AX}$ distribution pattern of early spermatogenic cells.

226 Figures 6B-6C show that chronic exposure to DEP significantly decreased the proportion of 227 seminiferous tubules with the leptotene or zygotene spermatocyte but increased the proportion 228 of seminiferous tubules with the pathytene or diplotene spermatocyte, strongly suggesting a 229 delayed transition from zygotene to pathytene induced by chronic DEP exposure.

\section{Chronic exposure to DEP alters the testicular gene expression profile}

232 To thoroughly determine the effect of chronic DEP exposure on the testis, we additionally 233 profiled the testicular gene expression of Vehicle- or DEP-exposed mice through deep RNA 234 sequencing. After the quality control, alignment to the mouse genome, and assembling of 235 transcripts, 18598 transcripts were identified. The volcano plot (Figure 7A) reveals that there 236 are 80 genes differentially expressed in the testes of DEP-exposed mice versus those of 237 Vehicle-exposed mice ( $p$ value of FDR $<0.05$ and fold change $<0.5$ or $>2$ ): 56 genes were 238 under-expressed in the DEP- versus Vehicle-exposed testes, and 24 genes were over239 expressed in the DEP- versus Vehicle-exposed testes. The relative expression levels of these 240 differentially expressed genes are presented in Figure 7B. To identify the biological processes 241 that are influenced by the chronic DEP exposure, gene ontology (GO) enrichment analysis 242 using the 80 differentially expressed genes was performed. Figure 7C shows that $8 \mathrm{GO}$ terms 243 were significantly enriched, including our previously identified GnRH signaling pathway. 


\section{Discussion}

246 The male reproductive system is vulnerable to environmental pollution, and published studies

247 have increasingly demonstrated that it may be targeted by $\mathrm{PM}_{2.5}$ exposure. However, the 248 biological mechanism by which $\mathrm{PM}_{2.5}$ exposure disrupts the male reproductive system and thus 249 the male fertility has not yet been fully understood. In the present study, we show that chronic 250 exposure to DEP, an important source for ambient $\mathrm{PM}_{2.5}, 1$ ) impaired the fertility of male mice 251 but did not influence their libido; 2) decreased the count and motility of epididymal sperms; 3 ) 252 compromised the repair of meiotic DSBs and thus the meiotic progression during 253 spermatogenesis; and 4) massively altered the testicular gene expression profile including the $254 \mathrm{GnRH}$ signaling pathway. To our best knowledge, this is the first study showing that $\mathrm{PM}_{2.5}$ exposure compromises the male fertility through targeting the repair of meiotic DSBs and thus the spermatogenesis and also the first high-throughput data-based evidence for the implication

257 of the HPG axis in the impairment of male fertility by chronic $\mathrm{PM}_{2.5}$ exposure.

258 Epidemiological studies increasingly demonstrate that exposure to $\mathrm{PM}_{2.5}$ inversely correlates with semen quality and male fertility rate [4-8]. The present study corroborates the impairment of semen quality and thus male fertility in a mouse model. The sperm count, motility, and morphology collectively determine the semen quality and thus male fertility. Notably, the present

262 study shows that chronic exposure to DEP decreased the epididymal sperm count and motility 263 but did not increase the rate of abnormal sperm (Figure 2). The lack of effect on the rate of 264 abnormal sperm is supported by our present data showing that chronic exposure to DEP did not 265 increase the rates of stillbirth and absorption (Table 1), and also consistent with our [15] and 266 others' $[9,13,18,20]$ published data. However, in contrast to the very consistent published data 267 regarding the adverse effects of $\mathrm{PM}_{2.5}$ exposure on the sperm count and motility [9,13- 
$15,17,18,20,21]$, there are several published studies showing that exposure to $\mathrm{PM}_{2.5}$ increases

269 the rate of abnormal sperm [14,17,21]. Although the reason for this confliction of published data

270 regarding the effect of $\mathrm{PM}_{2.5}$ exposure on the rate of abnormal sperm remains to be determined,

271 it somehow suggests a composition-dependency for this particular effect of $\mathrm{PM}_{2.5}$ exposure.

272 This also suggests that $\mathrm{PM}_{2.5}$ exposure impact the sperm count, motility and the rate of 273 abnormal sperm probably through different mechanisms.

274 In the present study, we demonstrate that chronic exposure to DEP did not impact the 275 insemination capacity of male mice neither the time taken for the insemination. To our 276 knowledge, this is the first evidence for that $\mathrm{PM}_{2.5}$ exposure decreases the male fertility rate not 277 through impact on the sexual behaviors. Thus, these results add massive support for the crucial 278 role of decreased semen quality in the induction of male infertility by $\mathrm{PM}_{2.5}$ exposure.

279 To date, although there is considerable evidence supporting the adverse effect of $\mathrm{PM}_{2.5}$ exposure on the spermatogenesis and thus the semen quality, the biological mechanism 281 remains elusive. The present study demonstrates that chronic exposure to DEP reduced 282 advanced spermatogenic cells but not early stages of germ cells such as spermatogonia in the 283 Stage VII seminiferous tubules. This is consistent with several published studies [9,15]. Given 284 that the advanced spermatogenic cells are produced in turn from the primary spermatocytes 285 through meiosis, these data strongly suggest that exposure to $\mathrm{PM}_{2.5}$ disturbs spermatogenesis 286 probably through targeting the spermatogenetic meiosis. The meiotic recombination between 287 homologous chromosomes through the programmed homologous pairing and formation and 288 repair of DSBs is a rich source of diversity in a population, and the meiotic progression is 289 regulated primarily using the recombination checkpoint that monitors meiotic recombination 290 during meiosis and blocks the entry into metaphase I if recombination is not properly processed. 291 The present study shows that chronic exposure to DEP markedly influenced the processing of 
292 meiotic recombination, particularly the repair of meiotic DSBs, as evidenced by both the 293 increased mis-pairing of sex and other chromosomes (Figure 5C,D) and the increased DSBs 294 in pachytene spermatocytes (Figure $\mathbf{5 H}$ ). The present data showing that chronic exposure to 295 DEP significantly altered the proportion of different stages of seminiferous tubules (Figures 3D, 296 6B, and 6C) strongly support that the disruption of meiotic recombination by chronic DEP 297 exposure is toxicologically significant. To our best knowledge, this is the first study 298 demonstrating that chronic exposure to $\mathrm{PM}_{2.5}$ disrupts spermatogenesis through targeting the 299 meiotic recombination. Because a scientific framework for extrinsic factors to regulate the repair 300 of meiotic DSBs has not yet been established, the present study did not investigate the 301 molecular mechanism by which $\mathrm{PM}_{2.5}$ exposure impact the repair of meiotic DSBs. Nonetheless, 302 the present study provides a valuable model to establish the scientific framework for the 303 regulation of repair of meiotic DSBs by extrinsic factors.

304 Notably, although the initiation of spermatogenic meiosis (the transition from A to A1 305 spermatogonia) is better known to be regulated by extrinsic factors [22], the present study 306 suggests that chronic exposure to DEP may not impact the initiation of spermatogenic meiosis, 307 as evidenced by the normal formation of DSBs in the leptotene spermatocytes (Figure $\mathbf{5 G}$ ) of 308 DEP-exposed testes and the normal number of spermatogonia of DEP-exposed testes (Figure 309 4E). Along with the above-mentioned evidence for the disruption of repair of meiotic DSBs by 310 chronic DEP exposure, these results strongly suggest that the repair of meiotic DSBs is 311 precisely targeted for chronic DEP exposure to impair the spermatogenesis.

312 The present study is also the first one using high-throughput technique to thoroughly 313 document the effect of chronic DEP exposure on the testicular gene expression profile. Another 314 important finding in the present study is the implication of the HPG axis in the development of 315 adverse effects on the male reproductive system due to exposure to DEP by our testicular gene 
316 expression profiling. Our gene expression profiling analysis showed not only that the

317 Glycoprotein Hormones, Alpha Polypeptide (Cga), the shared alpha subunit of luteinizing 318 hormone (LH) and follicle stimulating hormone $(\mathrm{FSH})$, is the most remarkable under-expressed 319 gene (Figure 7A) but also that the $\mathrm{GnRH}$ signaling pathway is one of eight significantly320 enriched GO terms (Figure 7C). Targeting the HPG axis for $\mathrm{PM}_{2.5}$ exposure to evoke adverse 321 effects on the male reproductive system is consistent with our [15] and others' [23] published 322 studies. Notably, recent studies showed that $\mathrm{PM}_{2.5}$ exposure disrupts the testicular histology and 323 spermatogenesis through a reactive oxygen species (ROS)-dependent mechanism [12,17]. 324 However, our testicular gene expression profiling did not identify any ROS-related genes 325 differentially expressed in DEP- versus Vehicle-exposed testes.

326 Although the present study provides compelling evidence that long-term exposure to DEP 327 affects the male reproductive system by disrupting first meiosis, it has a range of important 328 limitations. This includes the time and dose-dependent data that we have not provided for any of 329 these adverse effects due to DEP exposure. Another limitation is that this study failed to 330 determine which protein or gene changes in meiosis caused meiosis abnormalities. Of course, 331 this in-depth discussion requires a deeper accumulation of expertise and more sensitive 332 technology. Furthermore, the present study did not provide any data on the causal relationship 333 between meiosis arrest and damage to spermatogenesis. Therefore, it is necessary to conduct 334 additional experiments to determine how the male reproductive system damage via affecting the 335 meiosis process due to DEP exposure. 


\section{Methods}

\section{Animals}

339 All procedures of this study were approved by the Institutional Animal Care and Use Committee 340 at Fudan University, and all the animals were treated humanely and with regard for alleviation of 341 suffering. C57BI/6J mice (male, 4-week-old) were purchased from the Animal Center of 342 Shanghai Medical School, Fudan University (Shanghai, China) and were housed in standard 343 cages with a $12-\mathrm{h}$ light/12-h dark cycle with temperatures of $18-25^{\circ} \mathrm{C}$ and relative humidity of $40-$ $34460 \%$. One week of acclimation was allowed before the intratracheal instillation of DEP.

346 Intratracheal instillation of DEP

347 DEP was obtained from the National Institute of Standards and Technology (SRM 2975; NIST, 348 Gaithersburg, MD, USA). They were kept away from direct sunlight at $4^{\circ} \mathrm{C}$ before use. To 349 perform intratracheal instillation, DEP was first suspended in sterile PBS. To minimize 350 aggregation, DEP suspensions were sonicated (Clifton Ultrasonic Bath, Clifton, NJ, USA) for 20 351 mins on the day of instillation and vortexed for 30 s before instillation. The prepared DEP 352 suspensions were then intratracheally instilled to mice as previously described with minor 353 modification. In brief, animals were first anesthetized with $3 \%$ of isoflurane and placed supine 354 with extended neck on an angled board. A Becton Dickinson 18 Gauge cannula was then 355 inserted via the mouse mouth into the trachea. DEP suspension $(20 \mu \mathrm{g} / 50 \mu \mathrm{l}$ in PBS) or PBS 356 only was intratracheally instilled using a sterile syringe followed by $150 \mu \mathrm{l}$ air bolus. The mouse 357 was transferred to a vertical hanging position with its head up for $5 \mathrm{~min}$ after the removal of 358 intubation catheter to ensure that the delivered material was maintained in the lung without 359 blocking the airways. The deposition and distribution of instilled material was verified by Evans 
360 Blue (data not shown). PBS or DEP suspension was instilled 3 times/week (on Mondays, 361 Wednesdays and Fridays) for around 7 months.

\section{Fertility parameters collection}

364 Male C57BI/6J mice (5-week-old) were subject to intratracheal instillation of PBS/DEP for 6 365 months, and their fertilities were assessed via an 18-day mating with normal age-matched 366 female C57BI/6J mice followed by a week of singly housing. PBS/DEP instillation was continued 367 during the whole treatment period (thus approximately 7 months in total). Vaginal sperm plug was checked twice every day, and the insemination capacity was assessed as the percentage of mated mice showing the presence of sperm plug, time taken for insemination was assessed as the time spend between the start of mating and the earliest presence of sperm plug and the impregnation capacity was assessed as the percentage of pregnant mice after 18-day mating.

\section{Sperm count and motility analysis}

374 Sperm counting and motility analysis were conducted as previously described [24]. In brief, the 375 left epididymis was placed in $1 \mathrm{ml}$ normal saline, and then 6 deep cuts were made in each cauda 376 with micro-scissors to release sperms into the media at $35^{\circ} \mathrm{C}$ for $10 \mathrm{~min}$. The suspensions were 377 then filtered with nylon mesh (pore size of $70 \mu \mathrm{m}$ ) and stained with Papanicolaou (containing 378 formalin). The numbers and motility of sperm were then analyzed using a CASA system (IVOS

379 II Sperm Analyzer, Hamilton). At least 200 sperms from each sample were counted to assess 380 the abnormal sperm percentage. 

collection

384 On the day of experiment, after measurement of their body weight, all the mice were euthanized 385 and their blood was harvested from the orbital venous plexus. Fresh isolated testes, epididymis 386 and seminal vesicles were weighted, fixed in $4 \%$ paraformaldehyde for morphological analysis 387 and/or snap-frozen in liquid nitrogen and then stored at $-80^{\circ} \mathrm{C}$ for further use.

388 The testicular histology and spermatogenetic parameters were analyzed as described 389 previously [15]. In brief, freshly separated testicles were fixed in Bouin fixation fluid, dehydrated 390 and then embedded in paraffin. $5 \mu$ m-thick sections were prepared and stained with 391 hematoxylin-eosin. Pictures of all testicular tissues covering two successive sections of each 392 testicle were assessed by a pathologist who was blind to the sample grouping. All the 393 convoluted tubules in each sample section were used to assess Sertoli cell vacuolization, 394 tubules with deranged cell layer, percentage of stage VIII and VII seminiferous tubules. 4 stage 395 VII seminiferous round tubules (the ratio of long to short axis diameter < 1.2) of the first 396 observed field from each sample were used to assess the wall thickness, diameter, Sertoli cell 397 number, total germ cell/Sertoli cells, spermatogonia/Sertoli cells, pachytene 398 spermatocytes/Sertoli cells and round spermatids/Sertoli cells.

\section{Meiotic prophase cell spreading and immunofluorescence staining}

401 Spreads of spermatocytes and immunofluorescence staining were performed as previously 402 described [25]. In brief, testes of these PBS/DEP-exposed mice were placed in hypotonic 403 extracts (50mM sucrose, $17 \mathrm{mM}$ sodium citrate, $30 \mathrm{mM}$ Tris in pH8.2, 2.5mm DTT, $1 \mathrm{mM}$ PMSF in $404 \mathrm{pH} 8.3$ and 5mM EDTA), incubated on ice for 20 minutes and minced in $100 \mathrm{mM}$ sucrose. The 
spermatocyte spreads were then prepared on a slide and solidified in $1 \%$ PFA containing $0.1 \%$ Triton $\mathrm{X}-100$. The slides were incubated overnight in a damp room, dried and washed in PBS and water containing Photoflo (Kodak, NY, USA). Samples were first blocked using 10\% donkey serum with $3 \% \mathrm{BSA}$, and then stained with primary antibodies $\mathrm{YH} 2 \mathrm{AX}$ (1:500, Abcam), SYCP3 (1:100, Abcam) or DMC1 (1:100, Abcam) overnight at room temperature. Alexa 488 donkey anti-rabbit (1:500, Molecular Probes) and Alexa 594 goat anti-mouse (1:200, Molecular Probes) were then used as the secondary antibodies. The slides were incubated in darkness for 1 hour at $37^{\circ} \mathrm{C}$, washed, mounted with Vecta shield cover slips (Vector Laboratories) and imaged using confocal microscope (Nikon N-STORM).

\section{RNA sequencing and gene ontology (GO) analysis}

5 testis samples from each group were collected for the deep RNA sequencing test. The transcriptome libraries were constructed according to the MGIEasy RNA Library Prep Set V3.0 (MGI). For each RNA library, 20 million clean reads were generated by BGISEQ 500(BGIShenzhen). After passing the base composition and quality tests, the sequence of adapter, high content of unknown bases and low-quality reads were removed. Differentially expressed genes were identified using cuffdiff with standard parameters and clustered by Genesis using a hierarchical clustering method. Go enrichment was analyzed using Metascape (metascape.org). A hypergeometric test was performed using the default parameters to adjust the $p$ value.

\section{Statistics}

All data are expressed as means \pm SEMs unless noted otherwise. Statistical tests were performed using one-way or two-way analysis of variance (ANOVA) followed by Bonferroni 
428 correction or unpaired student's $t$ test using GraphPad Prism (version 5; GraphPad Software, La 429 Jolla, CA, USA). The significance level was set at $p<0.05$.

430

\section{Conclusion}

432 In summary, the present results demonstrate that long-term exposure to DEP impact 433 spermatogenesis by disrupting meiotic prophase and thus impair the male reproductive function.

434 To the best of our knowledge, our study is the first to use meiosis mechanism to analyze the 435 reproductive system damage caused by DEP exposure, which will provide a new idea for the 436 research on the male reproductive system damage caused by air pollution. 


\section{List of abbreviations}

$439 \mathrm{PM}_{2.5}$, ambient fine particles; CAP, concentrated ambient $\mathrm{PM}_{2.5}$; DEP, diesel exhaust $\mathrm{PM}_{2.5}$;

440 ANOVA, analysis of variance; DSBs, double strand breaks; WHO, world health organization;

441 BTB, blood-testis barrier; ROS, reactive oxygen species; ER, endoplasmic reticulum; PAHs, 442 polycyclic aromatic hydrocarbons; HPG, hypothalamic-pituitary-gonadal; GnRH, gonadotropin443 releasing hormone; $\mathrm{LH}$, luteinizing hormone; $\mathrm{FSH}$, follicle-stimulating hormone

\section{$444 \quad$ Funding}

445 This work was supported by the Ministry of Science and Technology of China 446 (2018ZX10301403-005-001 to WL), the National Institutes of Health (R01ES024516 to ZY), the 447 American Heart Association (13SDG17070131 to ZY), the National Natural Science Foundation 448 of China (Grant No. 91643205 to HK, Grant No. 81270342 to YX and Grant no. 81571495 to RL) 449 and Innovation-Oriented Science and Technology Grant from NHC Key Lab. of Reproduction 450 Regulation(CX2017-07 to RL).

\section{$451 \quad$ Availability of data and materials}

452 The datasets during and/or analyzed during the current study are available from the 453 corresponding author on reasonable request.

\section{Authors' contributions}

455 WY, HP and SS acquired the data used in the present study. YX, WY, HP and FT analyzed and 456 interpreted the present results. $\mathrm{YX}, \mathrm{ZY}, \mathrm{RL}$ and $\mathrm{WL}$ drafted the manuscript. $\mathrm{YW}, \mathrm{MX}, \mathrm{MY}, \mathrm{ST}$ 457 and $\mathrm{HK}$ were also major contributors in writing the manuscript. All authors read and approved 458 the final manuscript.

\section{Acknowledgements}

$460 \quad$ Not applicable.

\section{$461 \quad$ Ethics approval}


462 Fudan University is an AAALAC accredited institution. All procedures of this study were 463 approved by the Institutional Animal Care and Use Committee (IACUC) at Fudan University, 464 and all the animals were treated humanely and with regard for alleviation of suffering.

465 Consent for publication

466 Not applicable.

467 Competing interests

468 The authors declare that they have no competing interests.

469 


\section{References}

471 1. Huang C, Li B, Xu K, Liu D, Hu J, Yang Y, Nie H, Fan L, Zhu W. Decline in semen

2. Virtanen HE, Jørgensen N, Toppari J. Semen quality in the 21(st) century. Nature Reviews Urology. 2017;14:120.

3. Bowe B, Xie Y, Li T, Yan Y, Xian H, Al-Aly Z. The 2016 global and national burden of diabetes mellitus attributable to PM2.5 air pollution. The Lancet Planetary Health. 2018;2:e301-e312.

4. Huang X, Zhang B, Wu L, Zhou Y, Li Y, Mao X, Chen Y, Wang J, Luo P, Ma J. Association of Exposure to Ambient Fine Particulate Matter Constituents With Semen Quality Among Men Attending a Fertility Center in China. Environmental Science \& Technology. 2019:5957-5965.

5. Lafuente R, García-Blàquez N, Jacquemin B, Checa MA. Outdoor air pollution and sperm quality. Fertility and Sterility. 2016;106:880-896.

6. Lao XQ, Zhang Z, Lau A, Chan TC, Chuang YC, Chan J, Lin C, Guo C, Jiang WK, Tam T, Hoek G, Kan H, Yeoh EK, Chang LY. Exposure to ambient fine particulate matter and semen quality in Taiwan. Occup Environ Med. 2018;75:148-154.

7. Wu L, Jin L, Shi T, Zhang B, Liu Y. Association between ambient particulate matter exposure and semen quality in Wuhan, China. Environment International. 2017;98:219228.

8. Zhou N, Jiang C, Chen Q, Yang H, Wang X, Zou P, Sun L, Liu J, Li L, Li L, Huang L, Chen H, Ao L, Zhou Z, Liu J, Cui Z, Cao J. Exposures to Atmospheric PM10 and PM102.5 Affect Male Semen Quality: Results of MARHCS Study. Environ Sci Technol. 2018;52:1571-1581.

9. Watanabe N, Oonuki Y. Inhalation of diesel engine exhaust affects spermatogenesis in growing male rats. Environ Health Perspect. 1999;107:539-544.

10. Cao X, Shen L, Wu S, Yan C, Zhou Y, Xiong G, Wang Y, Liu Y, Liu B, Tang X, Guo M, Liu D, Long C, Sun M, He D, Lin T, Wei G. Urban fine particulate matter exposure causes male reproductive injury through destroying blood-testis barrier (BTB) integrity. Toxicology Letters. 2017;266:1-12.

11. Cao X, Yan C, Liu D, Peng J, Chen J, Zhou Y, Long C, He D, Lin T, Shen L, Wei G. Fine particulate matter leads to reproductive impairment in male rats by overexpressing phosphatidylinositol 3-kinase (PI3K)/protein kinase B (Akt) signaling pathway. Toxicology Letters. 2015;237:181-190.

12. Liu B, Wu SD, Shen LJ, Zhao TX, Wei Y, Tang XL, Long CL, Zhou Y, He DW, Lin T, Wei GH. Spermatogenesis dysfunction induced by PM2.5 from automobile exhaust via the ROS-mediated MAPK signaling pathway. Ecotoxicol Environ Saf. 2019;167:161-168.

13. Liu J, Ren L, Wei J, Zhang J, Zhu Y, Li X, Jing L, Duan J, Zhou X, Sun Z. Fine particle matter disrupts the blood - testis barrier by activating TGF - $\beta 3 / \mathrm{p} 38$ MAPK pathway and decreasing testosterone secretion in rat. Environmental Toxicology. 2018;33:711-719. 
14. Liu X, Jin X, Su R, Li Z. The reproductive toxicology of male SD rats after PM 2.5 exposure mediated by the stimulation of endoplasmic reticulum stress. Chemosphere. 2017; 189:547-555.

15. Qiu L, Chen M, Wang X, Qin X, Chen S, Qian Y, Liu Z, Cao Q, Ying Z. Exposure to Concentrated Ambient PM2.5 Compromises Spermatogenesis in a Mouse Model: Role of Suppression of Hypothalamus-Pituitary-Gonads Axis. Toxicological Sciences. 2018:1-12.

16. Wei Y, Cao XN, Tang XL, Shen LJ, Lin T, He DW, Wu SD, Wei GH. Urban fine particulate matter (PM2.5) exposure destroys blood-testis barrier (BTB) integrity through excessive ROS-mediated autophagy. Toxicol Mech Methods. 2018;28:302-319.

17. Zhang J, Liu J, Ren L, Wei J, Duan J, Zhang L, Zhou X, Sun Z. PM2.5 induces male reproductive toxicity via mitochondrial dysfunction, DNA damage and RIPK1 mediated apoptotic signaling pathway. Sci Total Environ. 2018;634:1435-1444.

18. Zhou L, Su X, Li B, Chu C, Sun H, Zhang N, Han B, Li C, Zou B, Niu Y, Zhang R. PM2.5 exposure impairs sperm quality through testicular damage dependent on NALP3 inflammasome and miR-183/96/182 cluster targeting FOXO1 in mouse. Ecotoxicol Environ Saf. 2019;169:551-563.

19. Ren L, Zhang J, Wang J, Wei J, Liu J, Li X, Zhu Y, Li Y, Guo C, Duan J, Sun Z, Zhou X. Silica nanoparticles induce spermatocyte cell apoptosis through microRNA-2861 targeting death receptor pathway. Chemosphere. 2019;228:709-720.

20. Jeng HA, Yu L. Alteration of sperm quality and hormone levels by polycyclic aromatic hydrocarbons on airborne particulate particles. Environmental Letters. 2008;43:675-681.

21. Izawa H, Kohara M, Watanabe G, Taya K, Sagai M. Diesel exhaust particle toxicity on spermatogenesis in the mouse is aryl hydrocarbon receptor dependent. J Reprod Dev. 2007;53:1069-1078.

22. Griswold MD. Spermatogenesis. The Commitment to Meiosis. Physiological Reviews. 2016;96:1-17.

23. Hansen C, Luben TJ, Sacks JD, Olshan A, Jeffay S, Strader L, Perreault SD. The effect of ambient air pollution on sperm quality. Environ Health Perspect. 2010;118:203-209.

24. Qiu L, Qian Y, Liu Z, Wang C, Qu J, Wang X, Wang S. Perfluorooctane sulfonate (PFOS) disrupts blood-testis barrier by down-regulating junction proteins via $\mathrm{p} 38$ MAPK/ATF2/MMP9 signaling pathway. Toxicology. 2016;373:1-12.

25. Cai X, Li J, Yang Q, Shi Q. Gamma-irradiation increased meiotic crossovers in mouse spermatocytes. Mutagenesis. 2011;26:721-727. 
547 Table 1. Pregnancy outcomes. ${ }^{*} p<0.05$ versus PBS control, Chi-square test or student $t$ test.

\begin{tabular}{lccc}
\hline Index & PBS & DEP & p value \\
\hline Stillbirth rate & $1.12 \%$ & $5.45 \%$ & 0.12 \\
Absorption rate & $13.48 \%$ & $9.09 \%$ & 0.43 \\
Implantations per dam & $7.6 \pm 0.84$ & $7.7 \pm 1.3$ & 0.96 \\
Live fetuses per dam & $6.9 \pm 0.88$ & $6.7 \pm 1.26$ & 0.9 \\
Fetus weight (mg/fetus) & $4.43 \pm 0.68$ & $4.6 \pm 1.05$ & 0.9 \\
Placental weight (mg/placenta) & $0.88 \pm 0.1$ & $0.84 \pm 0.12$ & 0.79 \\
Uterus weight (mg) & $7.71 \pm 0.89$ & $7.68 \pm 1.55$ & 0.99 \\
\hline
\end{tabular}


549 Table 2. Reproductive organ weights of male mice after 6-month intratracheal instillation of 550 PBS/DEP. * $p<0.05$ versus PBS control, student $t$ test.

\begin{tabular}{cccc}
\hline Weight(g) & PBS & DEP & p value \\
\hline Body weight & $29.27 \pm 0.3698$ & $29.29 \pm 0.5804$ & 0.9771 \\
Testis & $0.2018 \pm 0.0064$ & $0.1834 \pm 0.0143$ & 0.2734 \\
Epididymis & $0.0889 \pm 0.0028$ & $0.0877 \pm 0.0021$ & 0.7393 \\
Seminal vesicle & $0.2606 \pm 0.0173$ & $0.2734 \pm 0.0146$ & 0.5782 \\
\hline
\end{tabular}

551 
553 Figure 1. Chronic exposure to DEP impairs the male fertility. A. Experimental scheme. B. 554 Insemination capacity of male mice after 6-month intratracheal instillation of PBS/DEP. $n=$ 555 10/group, versus PBS, repeated measures chi-square test. C. Time taken for insemination. $n=$ 556 10/group, versus PBS, repeated measures Kaplan-Meier survival analysis. D. Impregnation 557 capacity. $n=10$ /group, $p=0.06$ versus PBS, repeated measures chi-square test.

558 Figure 2. Chronic exposure to DEP impairs the quality of semen. A. Papanicolaou staining 559 images of sperm morphology. a, normal sperm; b-d, abnormal sperm head; e-f, abnormal sperm

560 tail. B. Sperm count in epididymis tissue of male mice after 6-month intratracheal instillation of 561 PBS/DEP. $n=10$ /group, ${ }^{*} p<0.05$ versus PBS, student $t$ test. C. Sperm motility of male mice 562 after 7-month intratracheal instillation of PBS/DEP. $n=10 /$ group, ${ }^{*} p<0.05$ versus PBS, student $t$

563 test. D. Abnormal sperm percentage of male mice after 7-month intratracheal instillation of 564 PBS/DEP. $n=10 /$ group, ${ }^{*} p<0.05$ versus PBS, student $t$ test.

565 Figure 3. Chronic exposure to DEP alters the testicular histology. A. Representative H\&E 566 staining images of seminiferous tubule morphology in testis of male mice after 6-month 567 intratracheal instillation of PBS/DEP. Yellow arrow: derangement of the cell layers of 568 seminiferous tubules; Red arrow: Sertoli cell vacuolization. B. Proportion of Sertoli cell 569 vacuolization based on H\&E images of testis. C. Proportion of seminiferous tubules with 570 deranged cell layer. D. Percentage of stage VIII seminiferous tubules. E. Percentage of stage 571 VII seminiferous tubules. $n=10 /$ group, ${ }^{*} p<0.05$ versus PBS, student $t$ test.

572 Figure 4. Chronic exposure to DEP results in a loss of advanced spermatogenic cells in 573 Stage VII seminiferous tubules. A. Stage VII seminiferous tubule wall thickness. B. Stage VII 574 seminiferous tubule diameter. C. Sertoli cell number/Stage VII seminiferous tubule. D. Total 
575 germ cells/Sertoli cells. E. Spermatogonia/Sertoli cells. F. Pachytene spermatocytes/Sertoli cells.

576 G. Round spermatid/Sertoli cells. $n=10 /$ group, ${ }^{*} p<0.05$ versus PBS, student $t$ test.

577 Figure 5. Chronic exposure to DEP impairs the repair of meiotic double strand breaks

578 (DSBs). A. Double immunofluorescence images of surface-spread chromatin preparations of 579 PBS/DEP-treated mice testes. Synapses of the homologous chromosome were observed by 580 labeling SYCP3(red), a lateral element of the synaptonemal complex, and the initiation and 581 repair of programmed DSB was observed by labeling $\mathrm{YH} 2 \mathrm{AX}$ (green). White arrow: autosomal 582 unfinished repair and sex vesicle formation failure. B. Representative double 583 immunofluorescence images of surface-spread chromatin preparations of DEP-treated mice 584 testes with labeling of SYCP3(red) and $\mathrm{yH} 2 \mathrm{AX}$ (green). a-d: $\mathrm{X}$ and $\mathrm{Y}$ chromosomes can't be 585 paired, and sex vesicle can't form. C. Percentage of abnormal XY body in pachytene stage cells. 586 D. Percentage of abnormal homologous chromosome in pachytene stage cells. E. Percentage 587 of spermatocytes in different stages. $n=6 /$ group, ${ }^{*} p<0.05$ versus PBS, student $t$ test. F. Double 588 immunofluorescence images of surface-spread chromatin preparations of PBS/DEP-treated 589 mice testes with labeling of SYCP3(red) and DMC1(green). G. Number of DMC1 foci in 590 zygotene stage cells. H. Number of DMC1 foci in pachytene stage cells. $n=3 /$ group, ${ }^{*} p<0.05$ 591 versus PBS, student $t$ test.

592 Figure 6. Chronic exposure to DEP disrupts the spermatogenesis through impact on the 593 repair of DSBs. A. IHC assay with $\mathrm{yH} 2 \mathrm{AX}$ specific antibody was performed on 18dpp testes. 594 Yellow arrow: diffused distribution pattern of $\mathrm{yH} 2 \mathrm{AX}$ expression represents leptotene/zygotene 595 spermatocytes. Red arrow: focused distribution pattern of $\mathrm{yH} 2 \mathrm{AX}$ expression represents 596 pachytene/diplotene spermatocytes. B. Proportion of seminiferous tubules with the 597 leptotene/zygotene spermatocytes. C. Proportion of seminiferous tubules with the 598 pachytene/diplotene spermatocytes. $n=10 /$ group, ${ }^{*} p<0.05$ versus PBS, student $t$ test. 
599 Figure 7. Chronic exposure to DEP alters the testicular gene expression profile. A.

600 Volcano plot of the identified testicular gene expressions. B. Heatmap of differentially expressed

601 genes in the testes of Vehicle/DEP-exposed mice. C. 8 significantly enriched GO terms of the 602 differential genes assessed by gene ontology (GO) analysis. $n=5$ /group. 
Figures

Figure 1

A

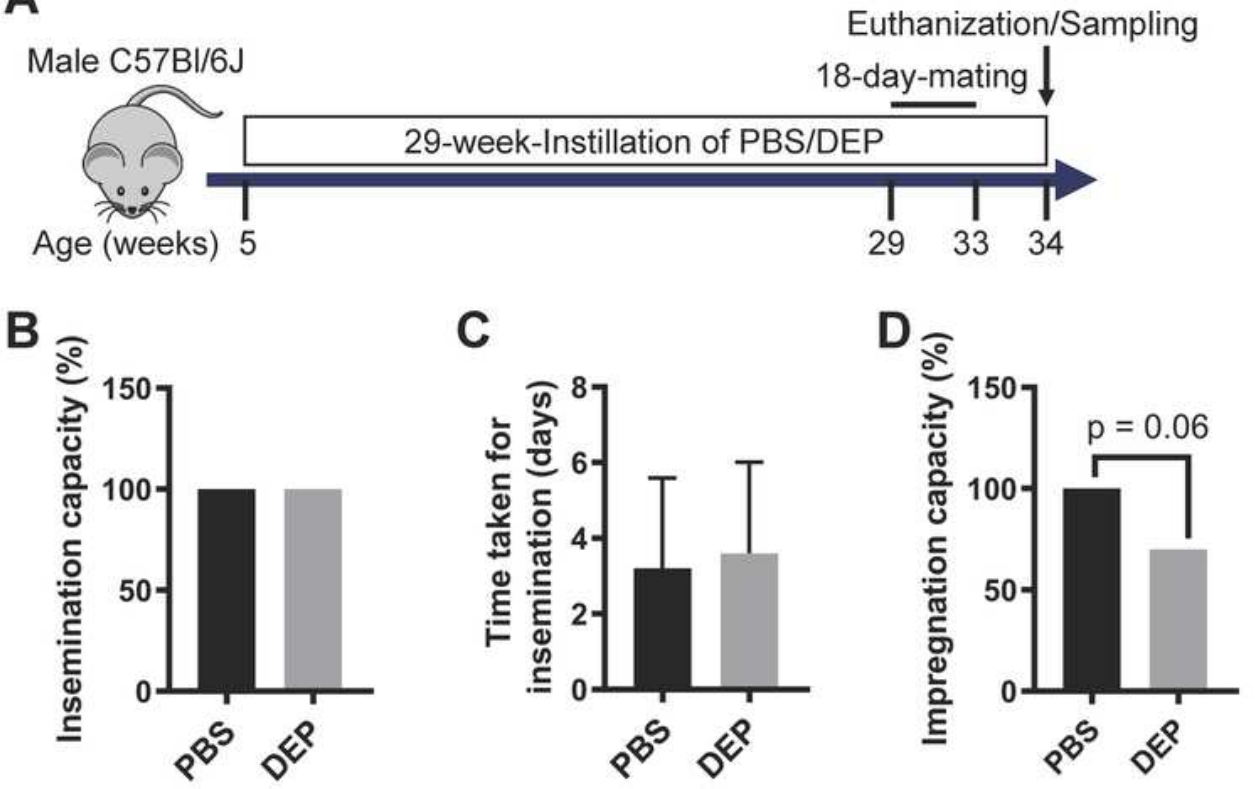

Figure 1

Chronic exposure to DEP impairs the male fertility. A. Experimental scheme. B. Insemination capacity of male mice after 6-month intratracheal instillation of PBS/DEP. $n=10$ /group, versus PBS, repeated measures chi-square test. $C$. Time taken for insemination. $n=10$ /group, versus PBS, repeated measures 
Kaplan-Meier survival analysis. D. Impregnation capacity. $n=10 /$ group, $p=0.06$ versus PBS, repeated measures chi-square test.

\section{Figure 2}
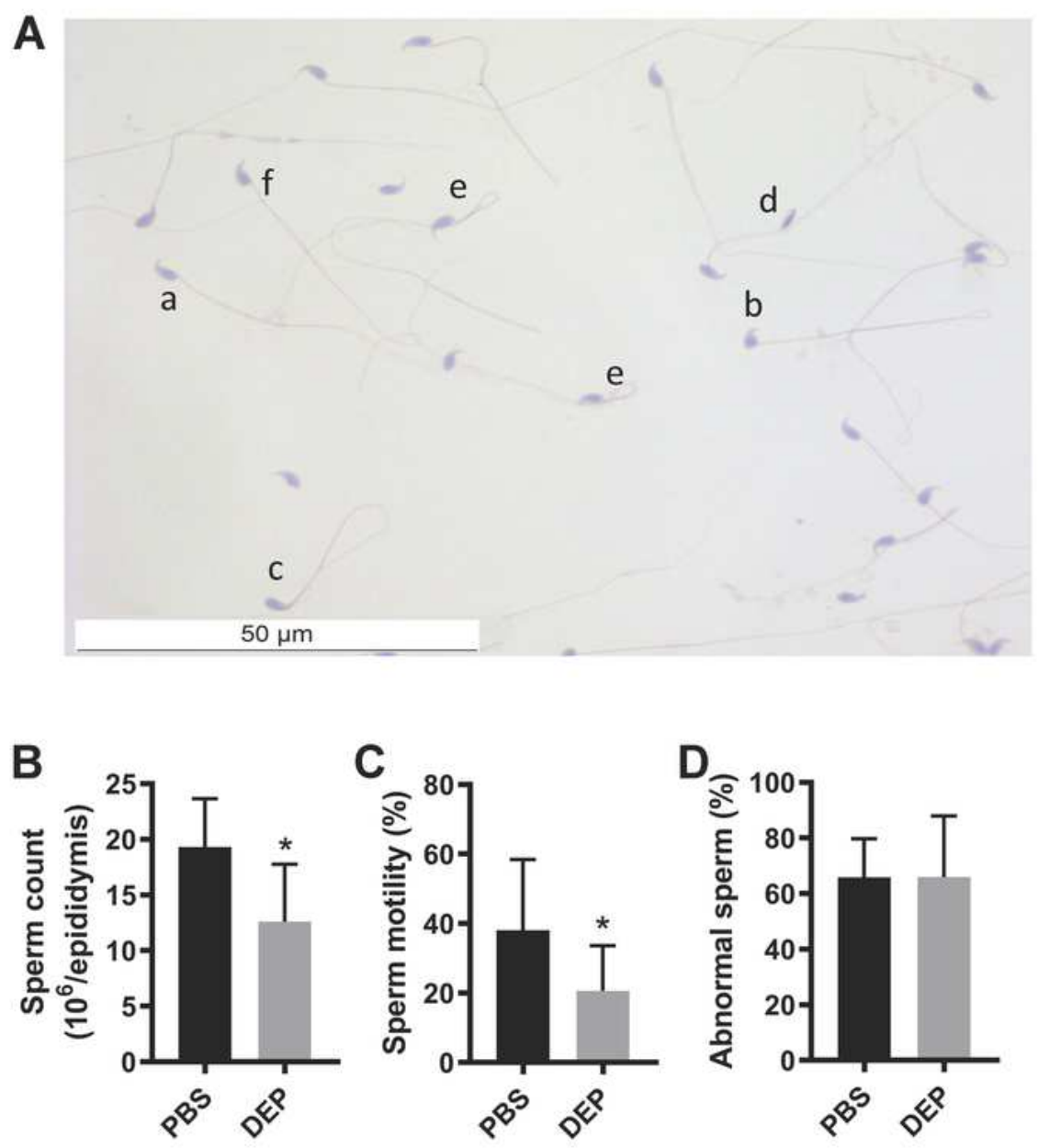

\section{Figure 2}

Chronic exposure to DEP impairs the quality of semen. A. Papanicolaou staining images of sperm morphology. a, normal sperm; b-d, abnormal sperm head; e-f, abnormal sperm tail. B. Sperm count in epididymis tissue of male mice after 6-month intratracheal instillation of PBS/DEP. $n=10$ /group, * $p<0.05$ 
versus PBS, student $t$ test. C. Sperm motility of male mice after 7-month intratracheal instillation of PBS/DEP. $n=10 /$ group, ${ }^{*} p<0.05$ versus PBS, student $t$ test. D. Abnormal sperm percentage of male mice after 7 -month intratracheal instillation of PBS/DEP. $n=10 /$ group, ${ }^{*} p<0.05$ versus PBS, student $t$ test.

\section{Figure 3}

A

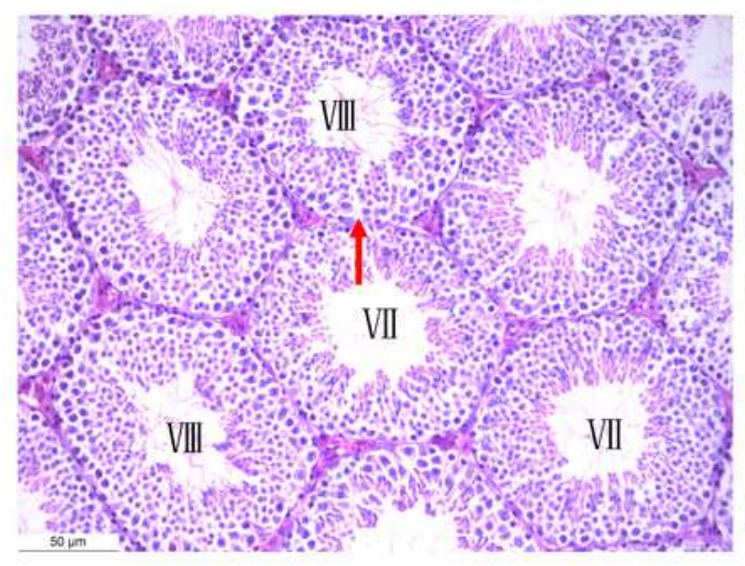

PBS
C

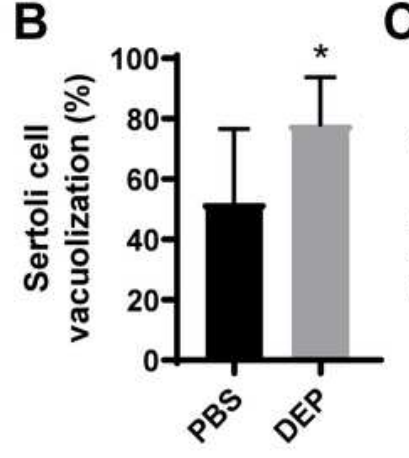

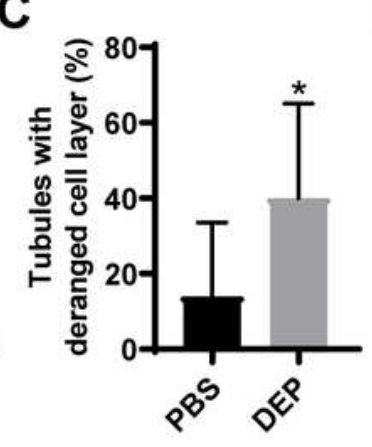

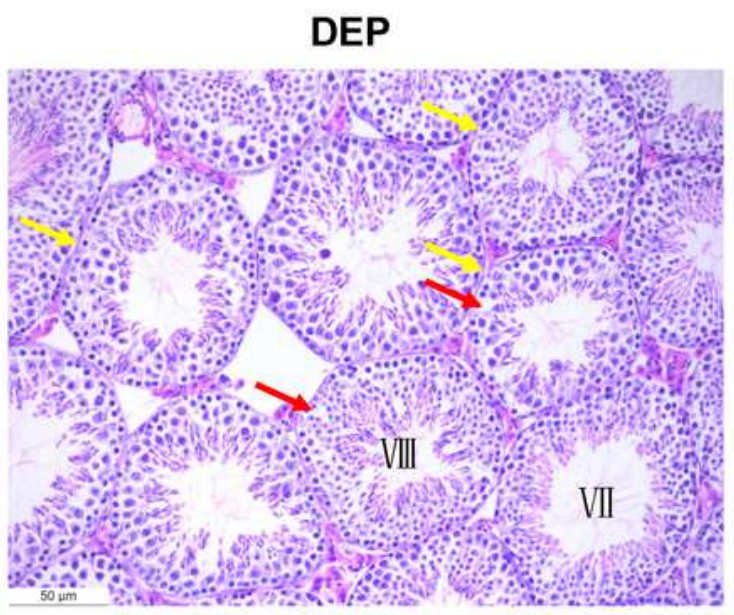

D

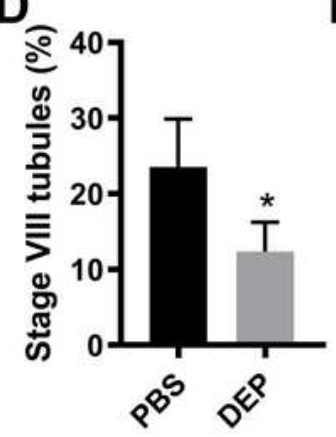

E

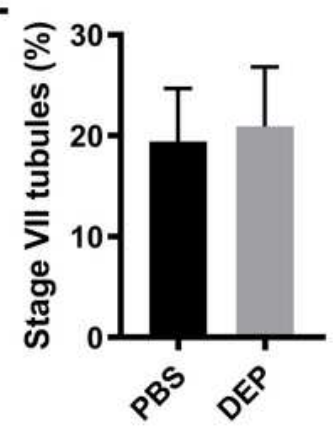

Figure 3

Chronic exposure to DEP alters the testicular histology. A. Representative H\&E staining images of seminiferous tubule morphology in testis of male mice after 6-month intratracheal instillation of 
PBS/DEP. Yellow arrow: derangement of the cell layers of seminiferous tubules; Red arrow: Sertoli cell vacuolization. B. Proportion of Sertoli cell vacuolization based on H\&E images of testis. C. Proportion of seminiferous tubules with deranged cell layer. D. Percentage of stage VIII seminiferous tubules. E. Percentage of stage VII seminiferous tubules. $n=10$ /group, ${ }^{*} p<0.05$ versus PBS, student $t$ test.

\section{Figure 4}
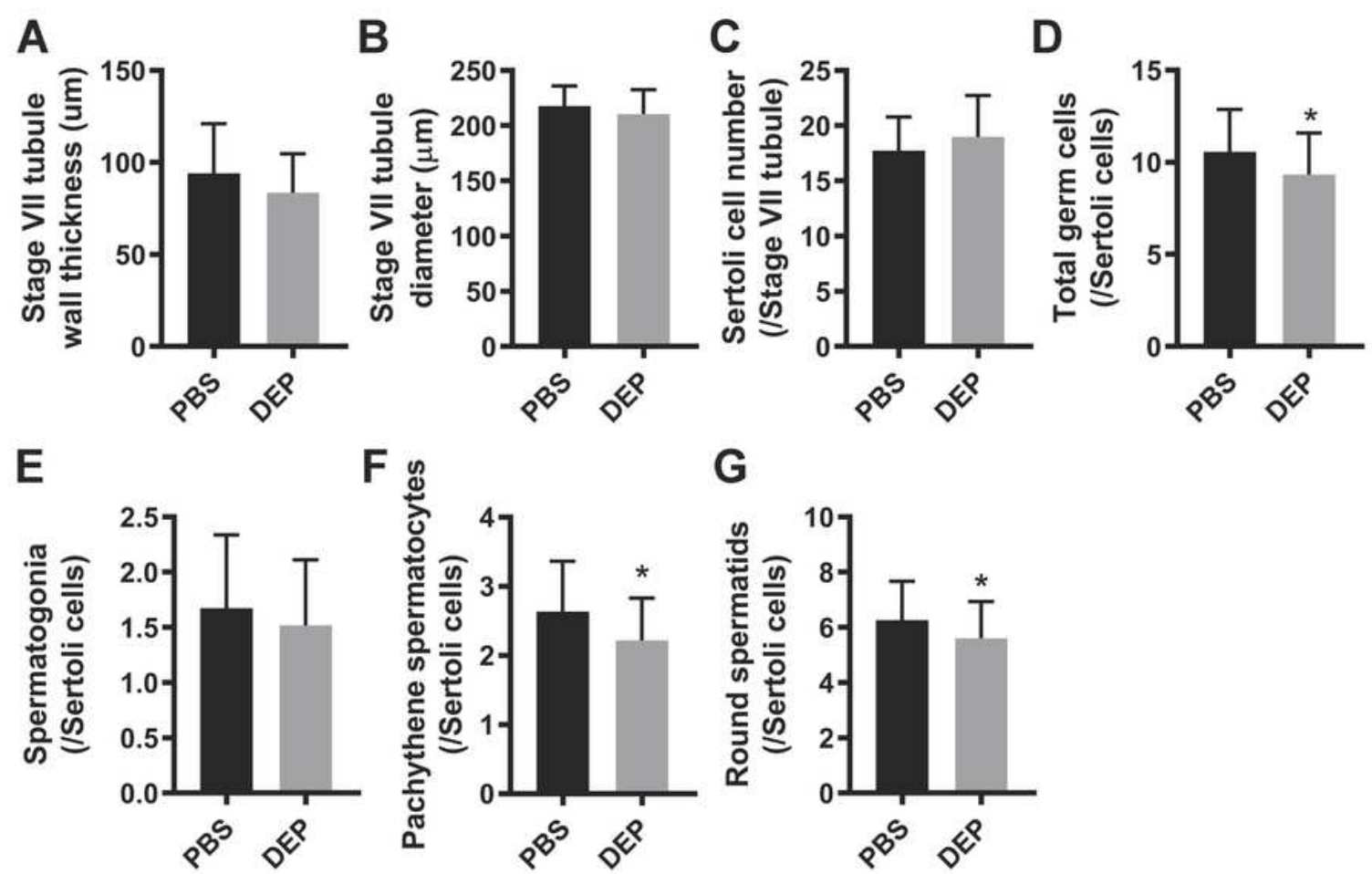

G

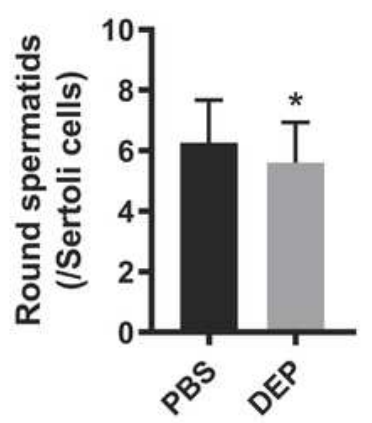

Figure 4 
Chronic exposure to DEP results in a loss of advanced spermatogenic cells in Stage VII seminiferous tubules. A. Stage VII seminiferous tubule wall thickness. B. Stage VII seminiferous tubule diameter. C. Sertoli cell number/Stage VII seminiferous tubule. D. Total germ cells/Sertoli cells. E.

Spermatogonia/Sertoli cells. F. Pachytene spermatocytes/Sertoli cells. G. Round spermatid/Sertoli cells. $n=10 /$ group, ${ }^{*} p<0.05$ versus PBS, student $t$ test.

\section{Figure 5}
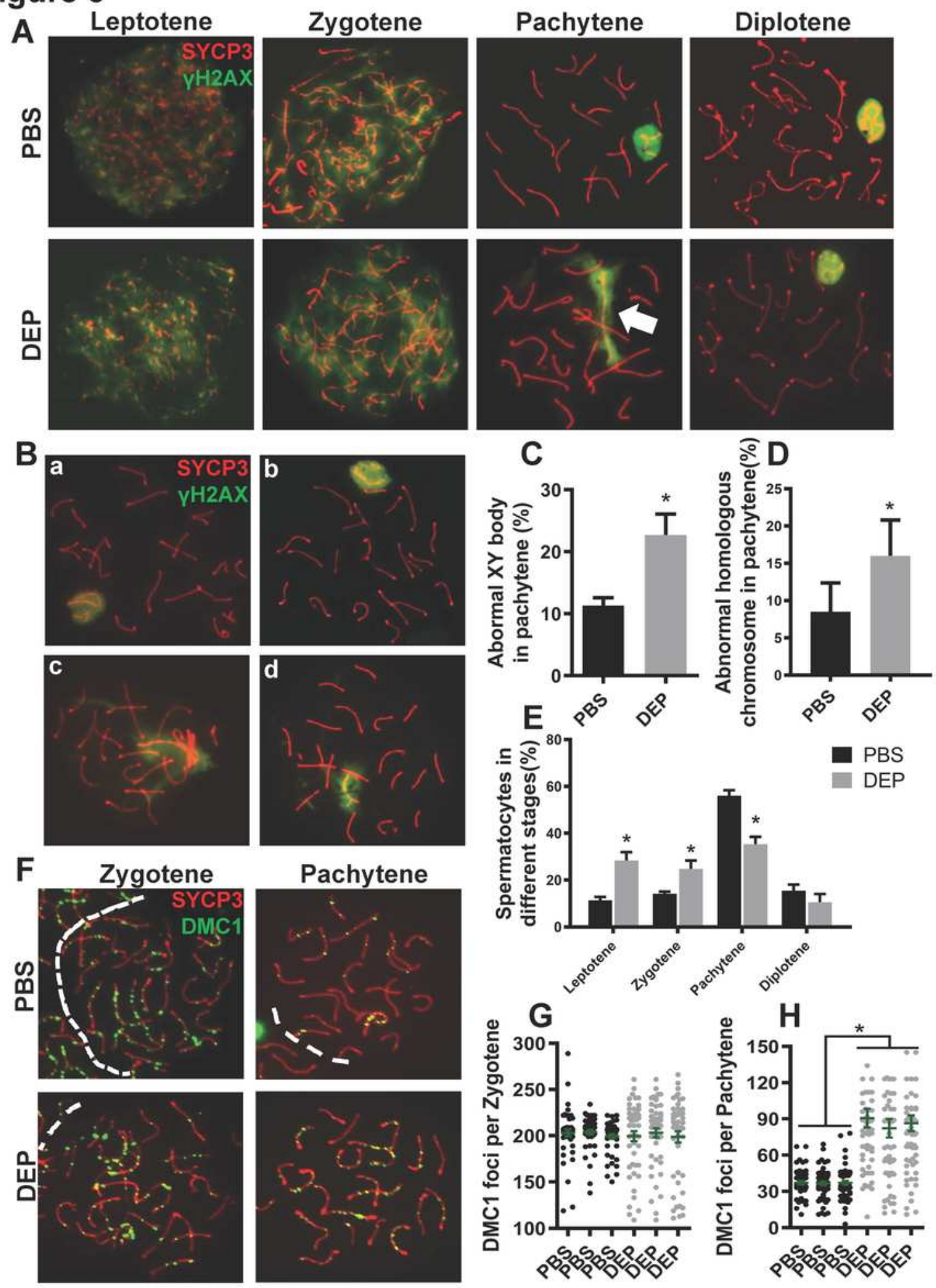

Figure 5 
Chronic exposure to DEP impairs the repair of meiotic double strand breaks (DSBs). A. Double immunofluorescence images of surface-spread chromatin preparations of PBS/DEP-treated mice testes. Synapses of the homologous chromosome were observed by labeling SYCP3(red), a lateral element of the synaptonemal complex, and the initiation and repair of programmed DSB was observed by labeling $\mathrm{YH} 2 \mathrm{AX}$ (green). White arrow: autosomal unfinished repair and sex vesicle formation failure. B. Representative double immunofluorescence images of surface-spread chromatin preparations of DEPtreated mice testes with labeling of SYCP3(red) and YH2AX (green). a-d: $X$ and $Y$ chromosomes can't be paired, and sex vesicle can't form. C. Percentage of abnormal XY body in pachytene stage cells. D. Percentage of abnormal homologous chromosome in pachytene stage cells. E. Percentage of spermatocytes in different stages. $n=6 /$ group, ${ }^{*} p<0.05$ versus PBS, student $t$ test. F. Double immunofluorescence images of surface-spread chromatin preparations of PBS/DEP-treated mice testes with labeling of SYCP3(red) and DMC1 (green). G. Number of DMC1 foci in zygotene stage cells. $\mathrm{H}$. Number of DMC1 foci in pachytene stage cells. $n=3$ /group, ${ }^{\star} p<0.05$ versus PBS, student $t$ test. 


\section{Figure 6}

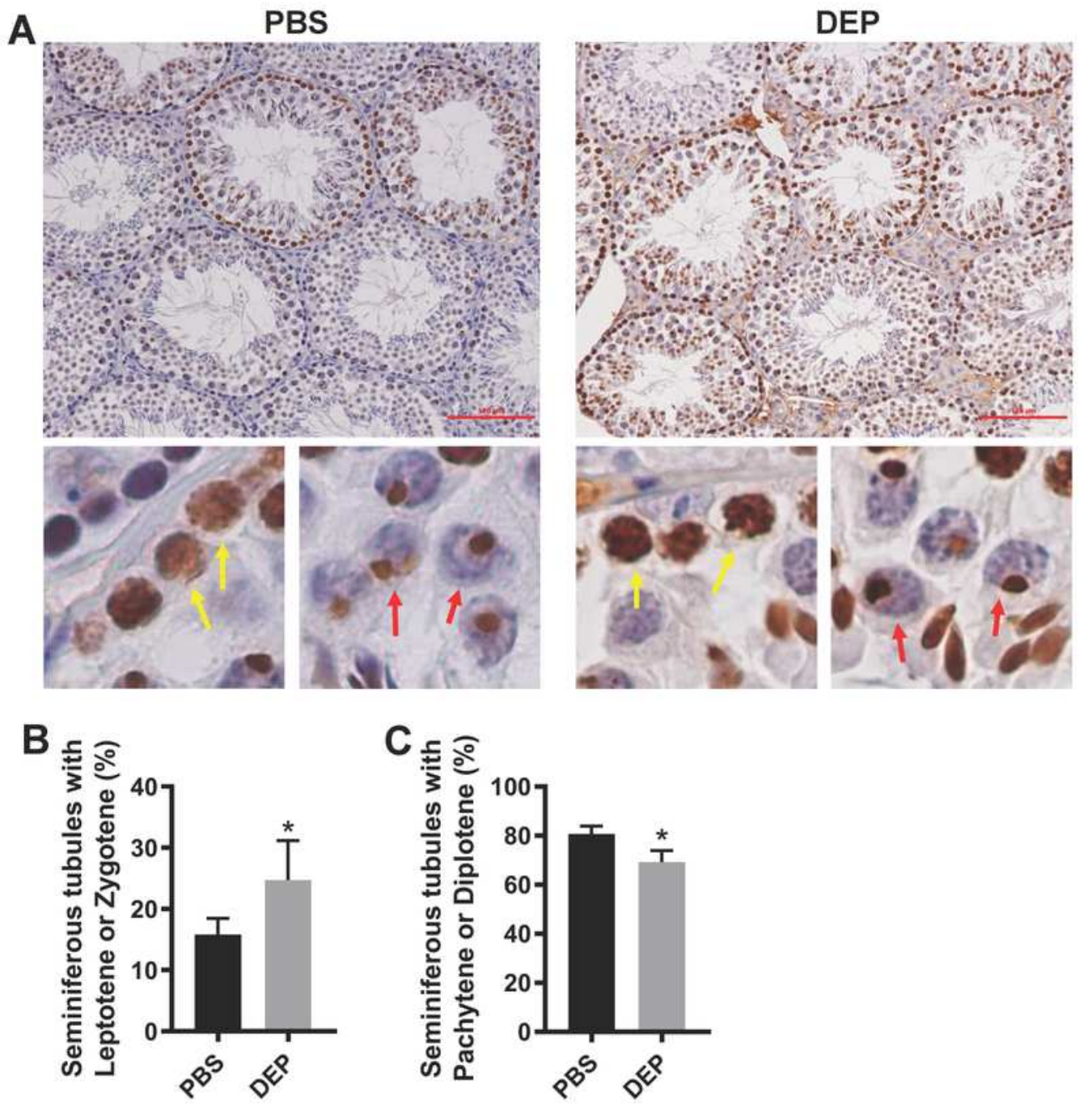

\section{Figure 6}

Chronic exposure to DEP disrupts the spermatogenesis through impact on the repair of DSBs. A. IHC assay with $\mathrm{YH} 2 \mathrm{AX}$ specific antibody was performed on 18dpp testes. Yellow arrow: diffused distribution pattern of $\mathrm{YH} 2 \mathrm{AX}$ expression represents leptotene/zygotene spermatocytes. Red arrow: focused distribution pattern of $\mathrm{yH} 2 \mathrm{AX}$ expression represents pachytene/diplotene spermatocytes. B. Proportion of 
seminiferous tubules with the leptotene/zygotene spermatocytes. C. Proportion of seminiferous tubules with the pachytene/diplotene spermatocytes. $n=10$ /group, ${ }^{*} p<0.05$ versus PBS, student $t$ test.

\section{Figure 7}

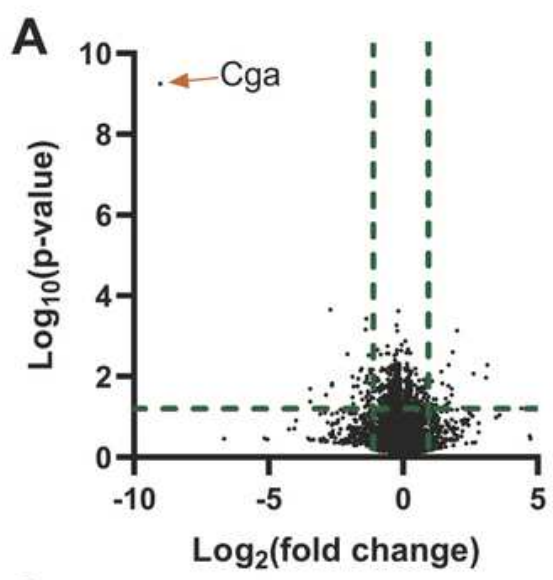

\section{B}
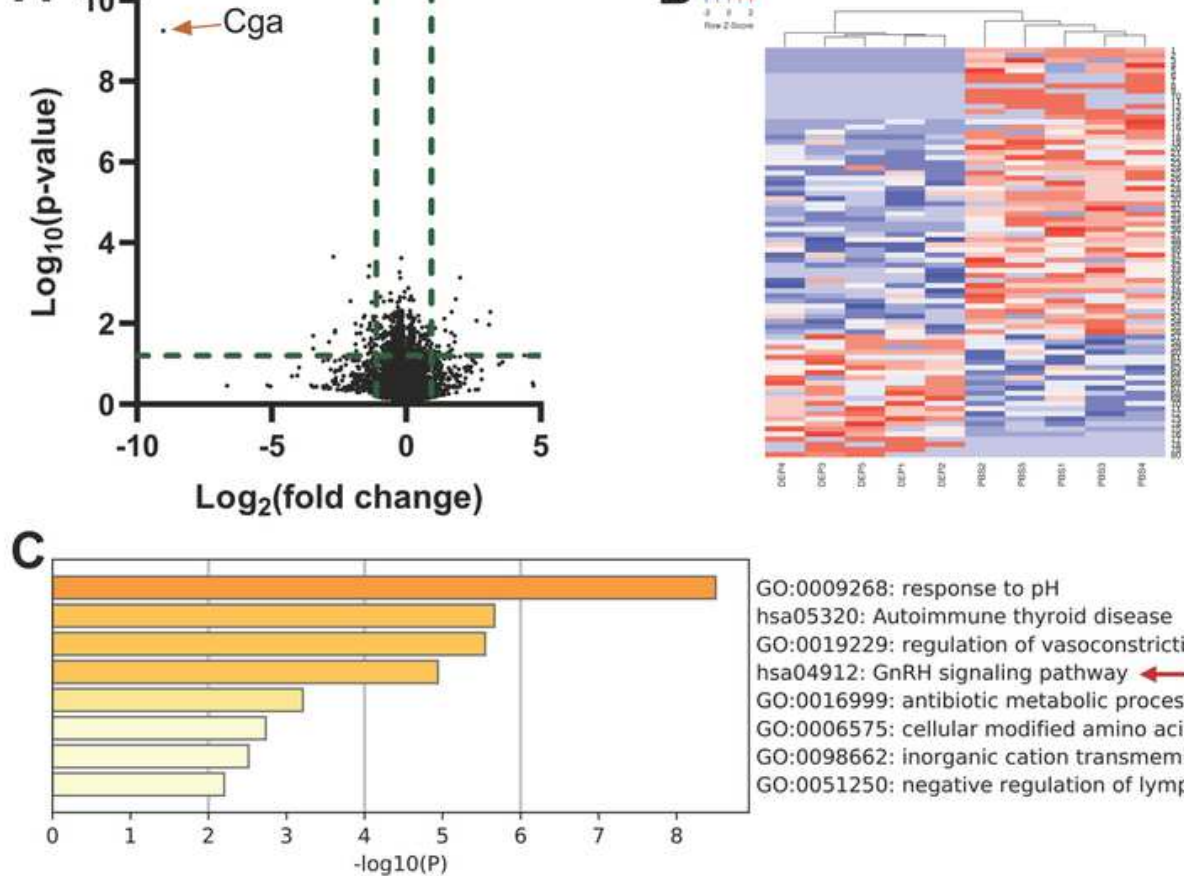

GO:0009268: response to $\mathrm{pH}$

hsa05320: Autoimmune thyroid disease

GO:0019229: regulation of vasoconstriction

hsa04912: GnRH signaling pathway $\longleftarrow$

GO:0016999: antibiotic metabolic process

GO:0006575: cellular modified amino acid metabolic process

GO:0098662: inorganic cation transmembrane transport

G0:0051250: negative regulation of lymphocyte activation

\section{Figure 7}

Chronic exposure to DEP alters the testicular gene 599 expression profile. A. Volcano plot of the identified testicular gene expressions. B. Heatmap of differentially expressed genes in the testes of Vehicle/DEP- 
exposed mice. C. 8 significantly enriched GO terms of the differential genes assessed by gene ontology (GO) analysis. $n=5 /$ group. 